\title{
Improved Geometry Modeling for High Fidelity Parametric Design.
}

\author{
James R. Gloudemans* \\ San Mateo, CA, 94403 \\ Robert McDonald ${ }^{\dagger}$ \\ California Polytechnic State University, San Luis Obispo, CA, 93407
}

\begin{abstract}
Advanced multidisciplinary physics-based design and analysis capabilities are required to pursue the revolutionary vehicle and technology concepts needed to meet the goals of the aerospace industry for the next 10-30 years. The multidisciplinary physics critical to advancing aerospace technology all have one common bond, the shape, size, and form of the underlying aircraft - its geometry. An aircraft shape is the natural starting point for multidisciplinary analysis and optimization (MDAO).

Vehicle Sketch Pad (VSP) is an aircraft geometry tool for rapid evaluation of advanced design concepts which was developed by NASA and is available to industry at large. VSP allows designers to express their design in terms of engineering parameters. The results of design choices are presented in real-time to the designer.

In this research, VSP has been extended in two major ways to improve the designer's ability to include high fidelity geometry based analysis in the design process. The first major improvement was the addition of a new high-quality surface meshing capability for VSP. The second major improvement was the addition of intuitive parametric representations of internal structure for VSP including the ability to convey that representation to appropriate structural modeling tools.
\end{abstract}

\section{Introduction}

Advanced multidisciplinary physics-based design and analysis capabilities are required to pursue the revolutionary vehicle and technology concepts needed to meet the goals of the aerospace industry for the next $10-30$ years.

An aircraft shape is the natural starting point for multidisciplinary analysis and optimization (MDAO). The outer mold lines and structural layout are the drivers for and interface between aerodynamics, structures, mass properties, and all the physics that impact a vehicle's performance.

Parameterization facilitates design and optimization by reducing the problem dimensionality and improving descriptive expressiveness. The aerospace industry and designers in particular have long described aircraft geometry parametrically; familiar quantities such as aspect ratio, taper ratio, sweep angle, and thickness to chord construct a common vocabulary for aircraft shape.

Vehicle Sketch Pad (VSP) is an aircraft geometry tool for rapid evaluation of advanced design concepts which was developed by NASA and is available to industry at large. ${ }^{1,2}$ Over the years, numerous aerospace groups have developed similar parametric geometry tools for aerospace design. ${ }^{3-8}$

A useful parametric geometry tool must not only depict the geometry, but it must translate the familiar description of an aircraft into a model which can be useful for engineering purposes such as CFD or FEA analysis.

*2309 Clipper Street.

${ }^{\dagger}$ Lockheed Martin Assistant Professor, Aerospace Engineering, One Grand Avenue, AIAA Member. 


\section{Surface Modeling \& Meshing}

VSP was originally developed to allow designers to express their design of the outer mold lines of an aircraft in terms of engineering parameters. The results of design choices are presented in real-time to the designer.

In order to provide real-time performance, the true wetted surface of the aircraft is not generated or updated with every design change. Instead, aircraft components are modeled and displayed independently but simultaneously; the wing and fuselage are both represented, but the wing-fuselage intersection is not calculated and the components are not trimmed at the intersection.

At any point in the design process, the designer may elect to calculate the component intersections and generate the outer mold lines of the vehicle. This true wetted surface can be used by VSP to perform a number of analysis tasks, or it can be output in a variety of file formats for analysis by external tools.

The shape and form of the wetted surface of an aircraft is the driver for its aerodynamic performance. As such, it naturally forms the starting point for drag buildup, panel codes, or supersonic wave drag analysis. Similarly, the surface representation generated by VSP can be used as the starting point for volume mesh generation to be used in CFD analysis.

The surface representation created by VSP can be used as input to a rapid prototyping machine or 3D printer. The process from concept to model can be very fast and very inexpensive. Rapid prototyping technologies are improving at an impressive pace; 3D printing of small scale wind tunnel models is now possible. Of course, the surface model output by VSP can also be used to create high quality illustrations and renderings of the design concept.

\section{II.A. Prior Approach}

There is an existing surface meshing algorithm in VSP used to compute trimmed volumes, wetted surface areas and mass properties. This method converts the Bezier surfaces into polygonal models. The triangles are then intersected using an octree to cull obvious non-intersecting cases. The intersected triangles are subdivided into smaller triangles ${ }^{9}$ and a ray cast method is used to remove interior triangles. This method is very fast but has several disadvantages. The resulting triangle quality is poor and may not be suitable many CFD and FEA codes. Also the tessellation is fixed and can not be refined in areas of interest or high curvature.

Although the new meshing algorithm has replaced this capability for all situations where high quality meshes are required, the old algorithm is still available. This algorithm is still very useful for quick analysis and tasks which do not require high fidelity such as the wetted area calculation used in a preliminary drag buildup.

\section{II.B. Current Approach}

\section{II.B.1. Background / Methods Considered}

Several meshing techniques were investigated to determine the best method to implement in VSP. The method chosen is detailed in the paper "EQSM: An efficient high quality surface grid generation method based on remeshing" by D. Wang et al. ${ }^{10}$

\section{II.B.2. Approach / Theory}

An aircraft configuration is constructed as normal in VSP (Figure 1). Code was added to VSP to skin the components with cubic Bezier surfaces and split them at sharp tangents. The Bezier surfaces are then intersected using a recursive splitting algorithm. ${ }^{11}$ And matched at the edges with trim curves. The trim curves are cubic Bezier curves in the parametric UV space of the surface. The matched trim curves are then tessellated based on the user defined grid density (Figure 2).

The grid density is specified by using point, line and box sources depicted in Figure 3. The desired edge length is defined for each source along with the affect radius. An algorithm has been implemented to automatically adjust the affect radius to assure smooth change of triangle sizes.

Next, an initial grid is generated in UV space as shown in Figure 4. This grid contains contain triangles of poor quality that do not match the specified grid density. The initial grid is remeshed and refined by using four tools: edge spitting, edge collapsing, edge swapping and point smoothing. 


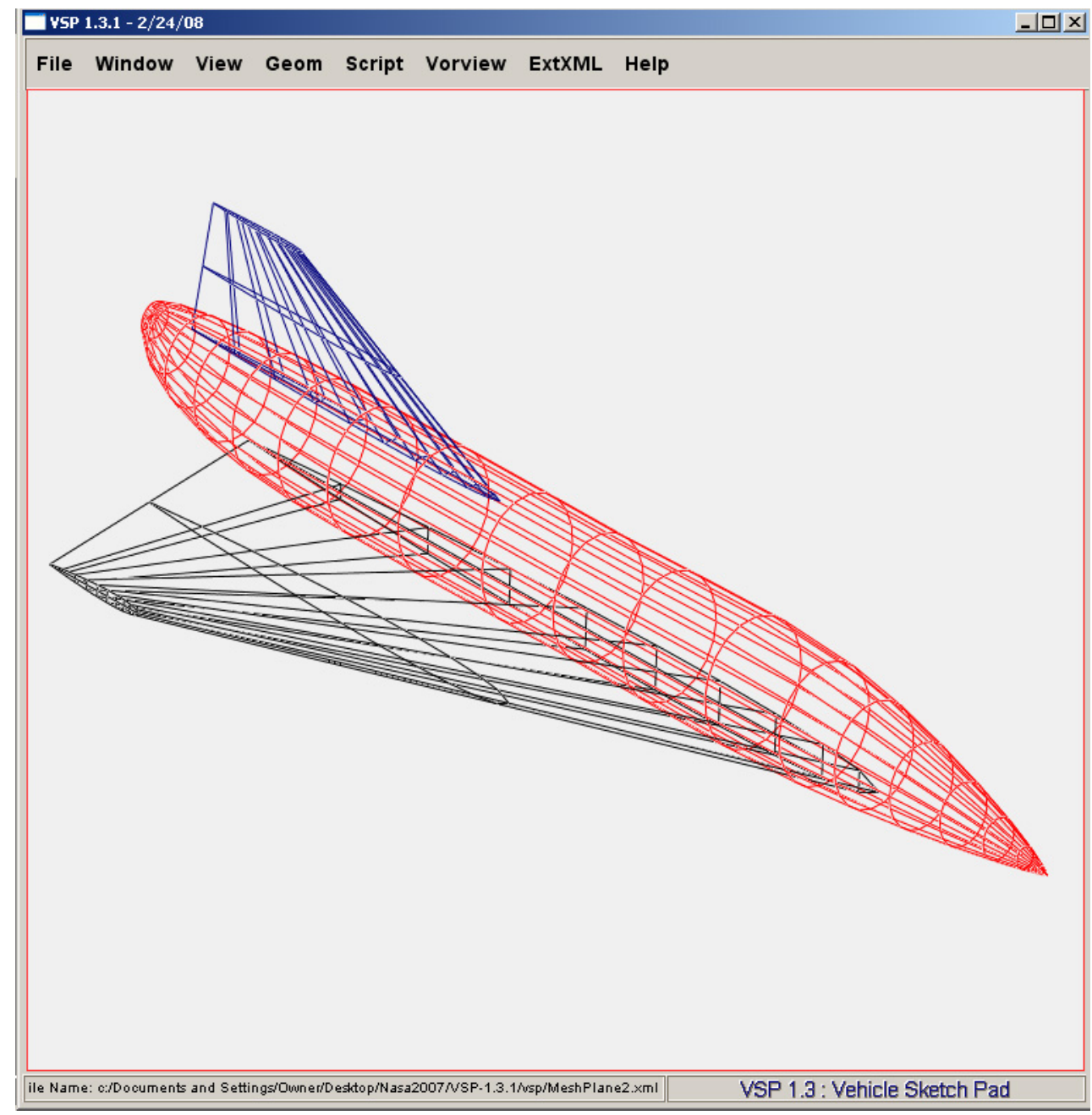

Figure 1. Aircraft geometry in VSP.

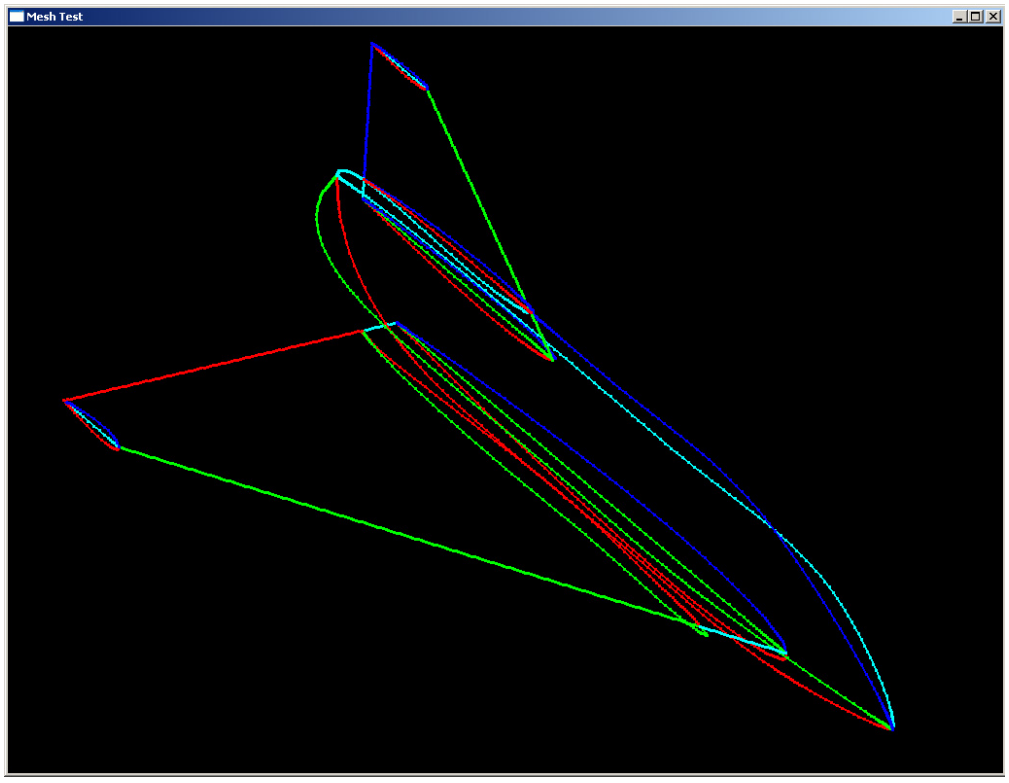

Figure 2. Grid points on patch boundaries. 


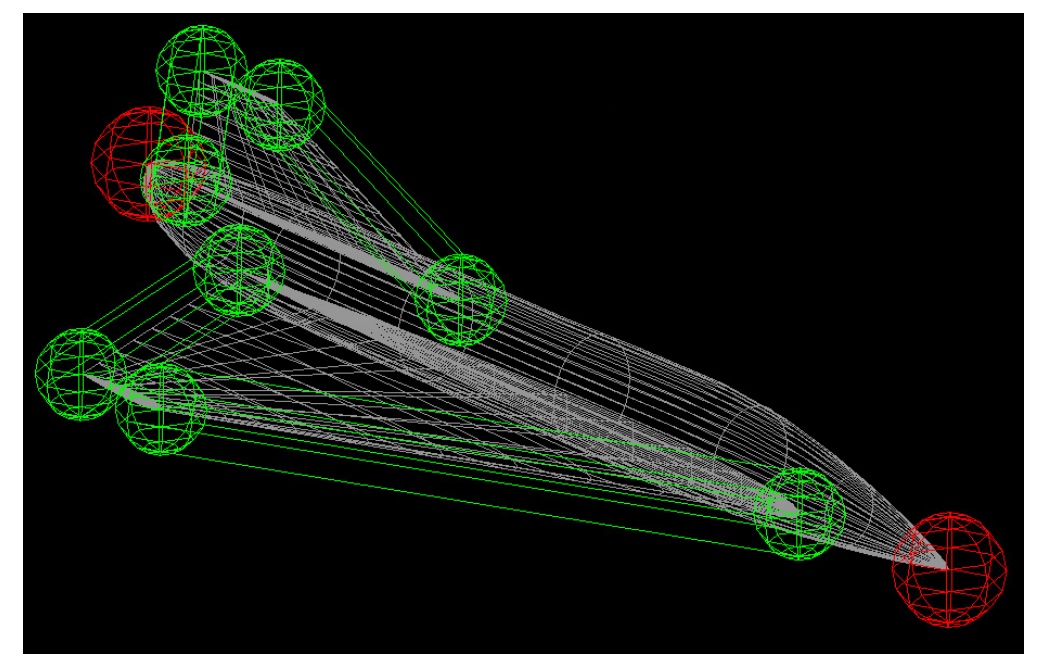

Figure 3. Grid density source controls.

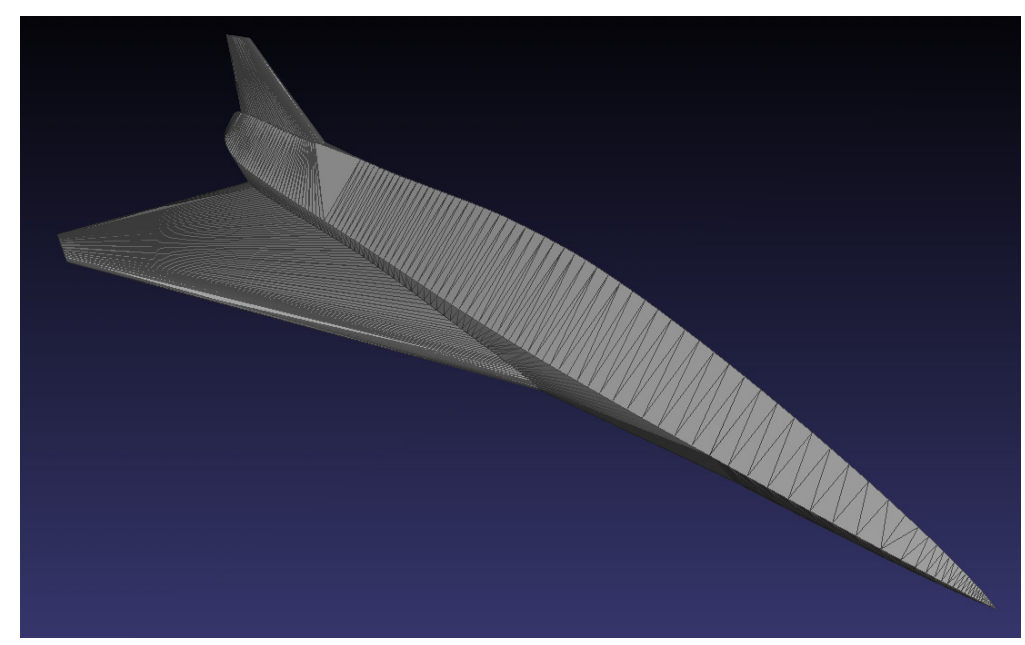

Figure 4. Low quality initial grid. 
Edge splitting involves finding all edges greater than the target length specified by the grid density. These edges are sorted and the longest ones are split first. The new node created by the split is projected back to the surface. Small edges are collapsed when the mesh topology is suitable. Edge swapping is done when it improves triangle quality. Finally, point smoothing is done by averaging the surround nodes in 3D space and projecting the resulting point back to the nearest surface UV point. During this remeshing process, the target edge length is computed from user defined grid density.

The final grid shown in Figures 5(a) and 5(b) contains 31,000 triangles. Approximately $0.1 \%$ of the triangles had an angle less than 20 degrees and most of them were located at the trailing edge of the wing tips.

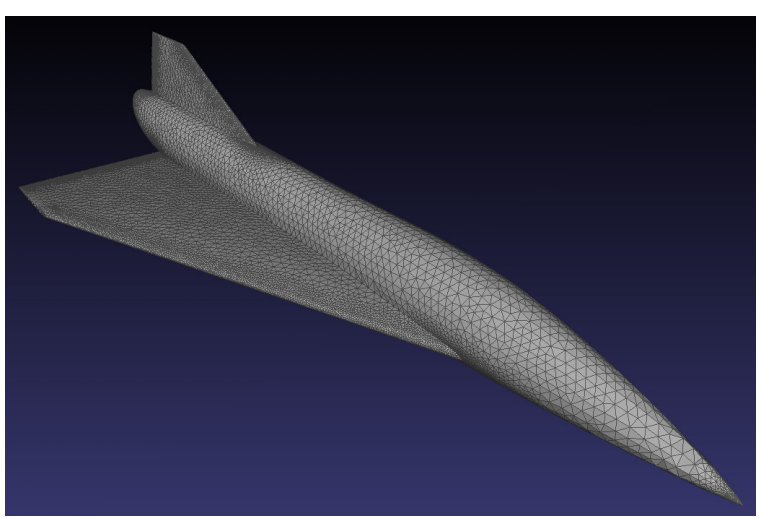

(a) Final mesh of aircraft geometry.

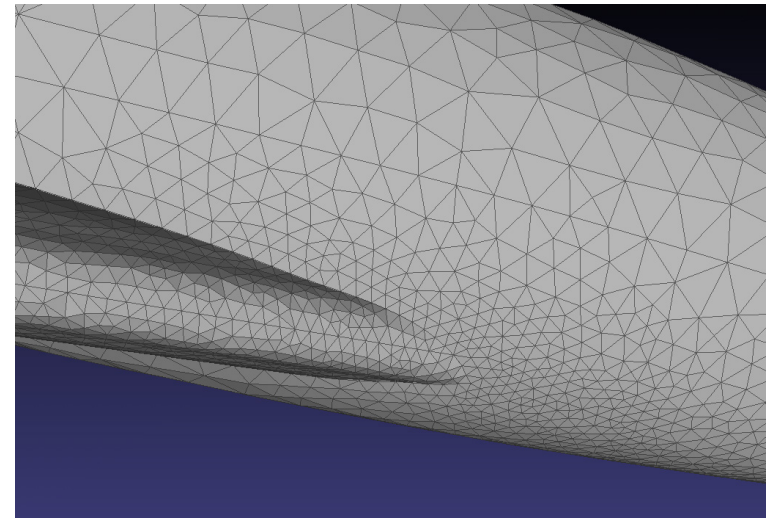

(b) Close up of wing-body junction for the final mesh.

Figure 5. High quality final grid.

\section{II.B.3. Parameterization}

Mesh grid density is defined and controlled by using point, line and box sources. These sources specify the desired edge length near the source. The radius of the source dictates the volume affected by that source. The target length is decreased by the square of the fractional distance away from the source. The source size may be automatically increased to prevent the edge length from changing more than $20 \%$ at source borders. The position of the sources is specified in the parameter space of the components. This allows the user to change the geometry without having to redefine sources (see Figure 6). Line and box sources require two points to specify location and size. To improve the speed of the mesh density specification, the meshing process is split into two parts: intersect and mesh. If the geometry does not change, only the mesh process is required to see changes in density. When the mesh density is satisfactory, the mesh can be exported in Nascart and stl formats.

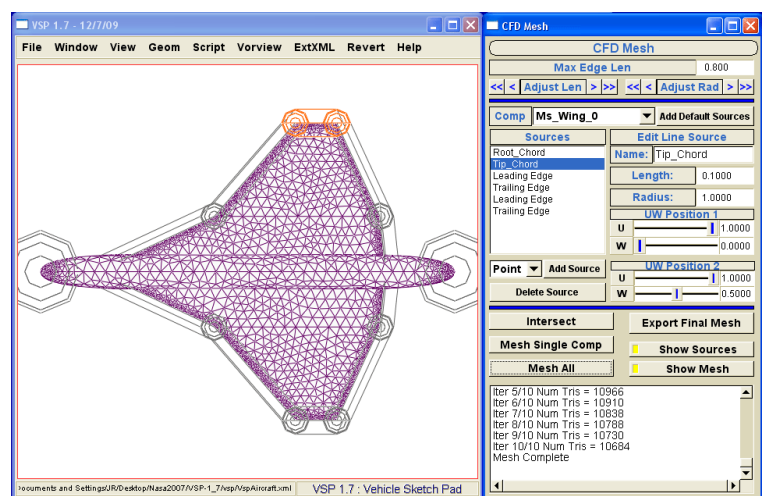

(a) Original geometry and mesh sourcing.

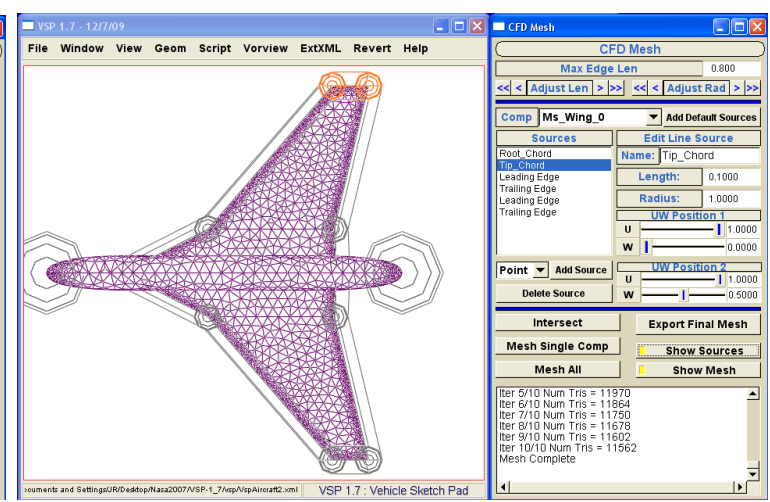

(b) Altered geometry with mesh sourcing.

Figure 6. Mesh sourcing control connected to parametric geometry. 
In normal operation, VSP does not display the intermediate steps to the user. The primary mesh control interface presented to the user is depicted in Figure 7. This interface allows the user to create and modify the grid density control sources.

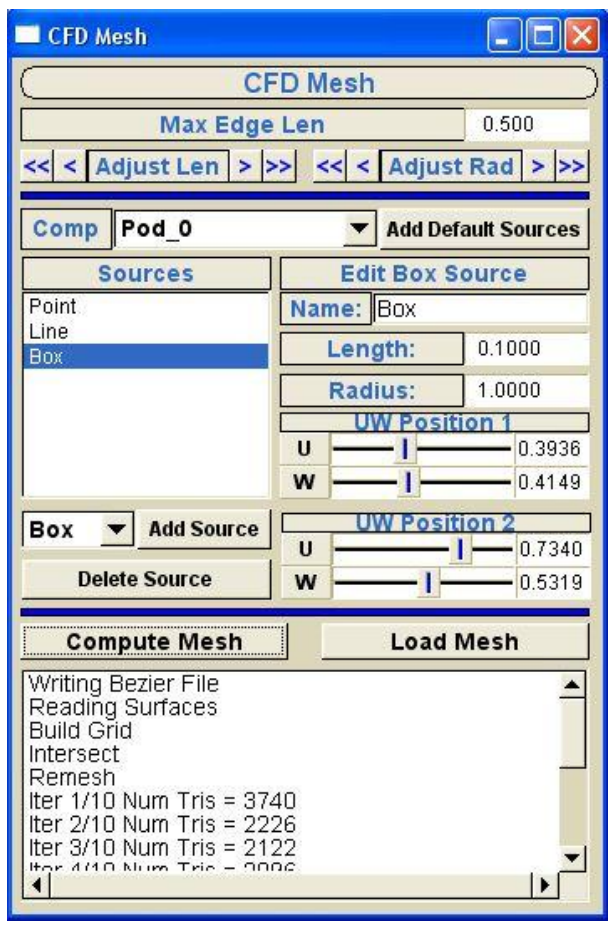

Figure 7. Meshing control GUI.

VSP has the capability to automatically create a default set of mesh control sources for each geometric component in the model. These default sources provide a reasonable "first-cut" at the mesh and provide a foundation for tweaking and customization. The default source arrangement for a complex aircraft is shown in Figure 8. In addition to the controls for the individual sources, the user can adjust some global parameters scaling mesh size and limiting the largest triangle in the mesh. These global parameters allow the user to quickly adjust a mesh as a whole, thereby creating a series of related meshes.

The high quality surface mesh resulting from the default source arrangement on the complex aircraft is included as Figure 9. This mesh consists of approximately 250,000 triangles.

\section{Internal Structure Modeling}

VSP has been extended to allow designers to express the internal arrangement of major structural components in addition to the outer mold lines of an aircraft. As with the outer mold lines, it was desired to present the design choices for the internal structure in real-time to the designer. Furthermore, it was necessary to develop a parametric representation of the aircraft structure that was both simple and intuitive for the designer to use, but still flexible and powerful enough to represent all the structural concepts the designer may envision.

Extending VSP to model the vehicle internal structural layout in addition to its wetted surface allows the designer to represent the complete vehicle design; the corresponding high quality mesh generation capabilities allows the complete vehicle design to be evaluated by high-fidelity physics-based models at a level unprecedented in the early stages of design. The mesh requirements for the structural analysis of an aircraft are somewhat different from those required for its external aerodynamics; VSP will support the needs of multiple disciplines. For example, quadrilateral elements are created where possible in the structural mesh while the aerodynamic mesh consists entirely of triangular elements.

The problem of providing a real-time representation of the internal structure was solved in a manner analogous to the wetted surface representation. The full-fidelity model of the internal structure is not updated with every design change; instead, an intuitive simplified model which represents the structure is 


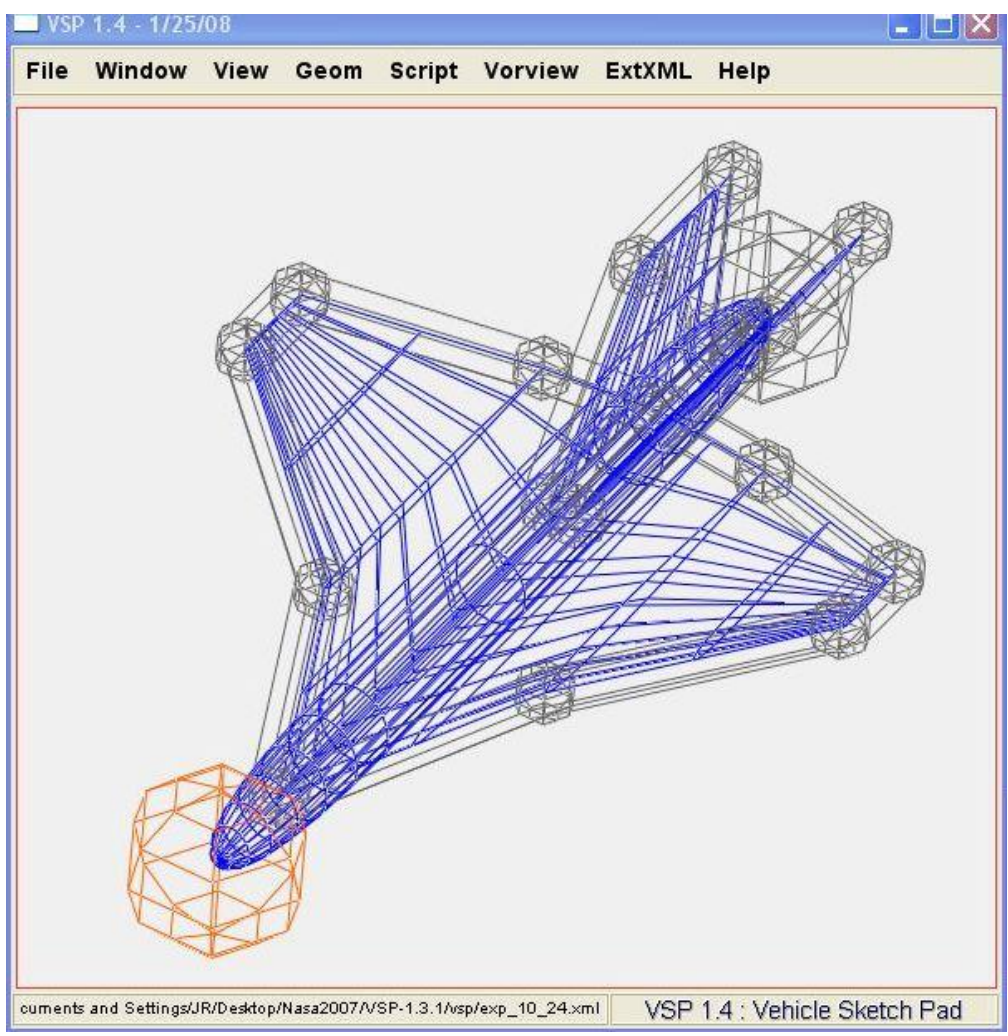

Figure 8. Default source arrangement for complex aircraft geometry.

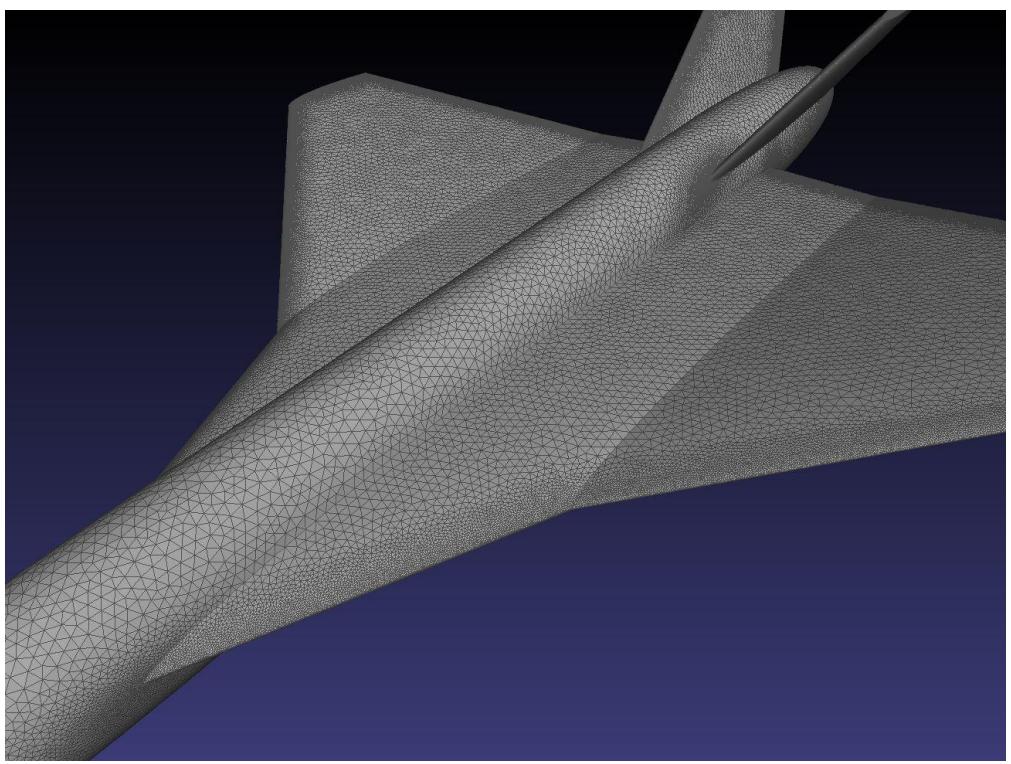

Figure 9. Surface mesh for complex aircraft geometry. 
presented to the designer. The structural modeling capabilities of VSP support specific parametric full depth structural components such as ribs and spars. These components combined with the aircraft skin to allow the modeling of many wing structures.

When the designer is satisfied with the internal structure, they can choose to generate a high fidelity model of the entire internal structure. Like the wetted surface, this true representation of the structure can be used by VSP to perform some analysis, or it can be output for analysis by external tools. The layout and arrangement of the internal structure as well as the component skins drive the structural and mass properties behavior of an aircraft. VSP can output the generated structural model to FEA packages such as NASTRAN and Calculix.

\section{III.A. Approach}

The FEA meshes are created by building a slicing plane for each rib and spar. These planes were intersected with each other and with the wing skin surfaces. The resulting border curves were tessellated based on the desired mesh density. The high quality meshing capabilities discussed in Section II are used to generate a mesh for each component. When appropriate, quad elements are generated to improved model fidelity.

\section{III.A.1. User Interface}

The user interface used to control the parametric structure definition is depicted in Figures 10 through 12. When defining a structural layout, the user would work with each of these interfaces to define the complete structure. The user can specify a parametrically varying skin thickness for a component using the interface depicted in Figure 10. Skin thickness is defined by using an arbitrary number of chordwise splice lines. The thickness along each splice line can be controlled at any location and the thickness is interpolated between lines.

Spanwise full-depth structural components such as wing spars are defined using the interface depicted in Figure 11. When components such as spars or ribs are defined, the VSP geometry window switches to a plan-view to clearly illustrate the concept being defined. Full-span and partial-span spars may be specified by the user. Spar angle may be controlled in terms relative to the wing panel, or in an absolute sense.

Chordwise full-depth structural components such as wing ribs are defined using the interface depicted in Figure 12. Ribs may be defined at any angle relative to the global coordinate system or relative to the wing panel itself. Consequently ribs may be easily laid out parallel to the flow, or perpendicular to a leading edge or a spar. When the user has finished laying out the structure, he or she may press the "Export FEA Mesh" button to generate the mesh and write out the FEA input files. The corresponding mesh for this stage of work is included in Figure 12.

\section{III.A.2. Results}

The VSP structure definition may be written out to a file for analysis by a finite element code. At present, files appropriate for analysis in NASTRAN and Calculix are produced. Calculix is an open source FEA code which has been selected for its price, ease of use, and availability to help debug the meshing process. An example analysis produced by Calculix is depicted in Figure 13. Also, the structural mass is computed based on user defined material thickness and density.

\section{Conclusions}

VSP, a parametric geometry tool for modeling aircraft, has been extended to improve support for high fidelity analysis for design. Two major improvements are documented in this paper. VSP now has the capability to generate high quality surface meshes of the modeled aircraft. These meshes provide a suitable starting point for high fidelity analysis such as CFD. VSP now also has the capability to model simple fulldepth wing structures and the structural properties of the wing skin. This model can be used to generate a FEA input file for structural analysis. 


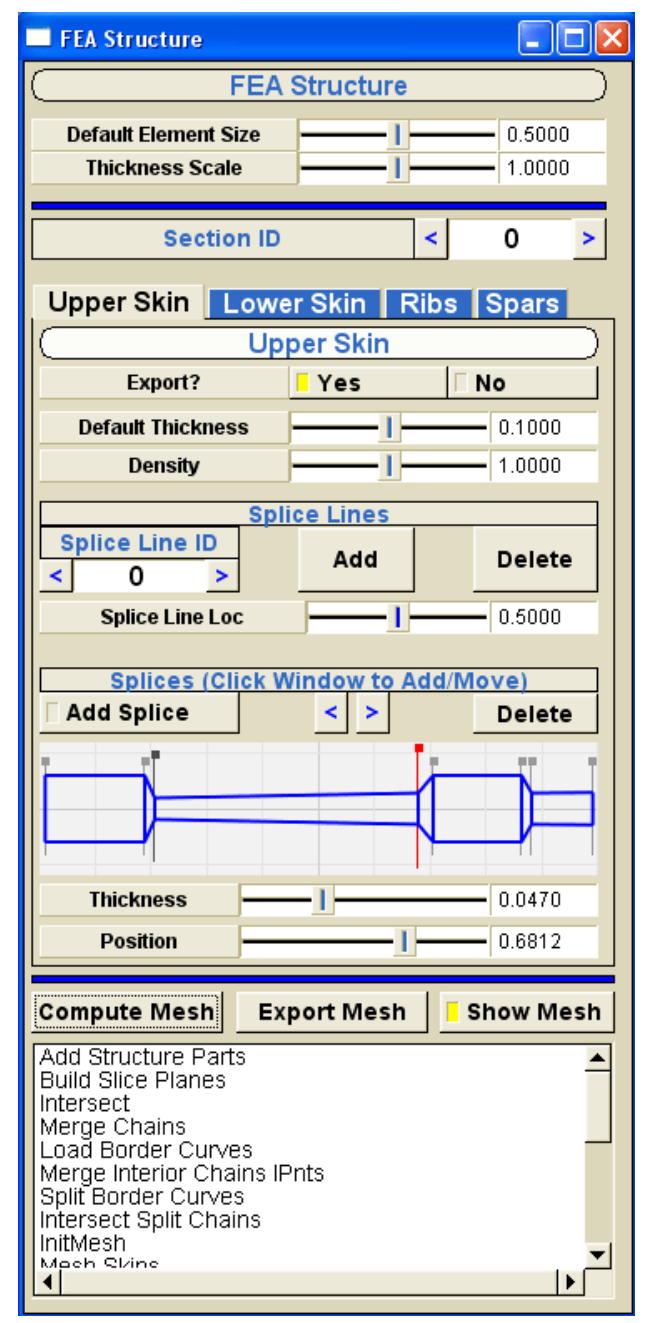

Figure 10. Structures control GUI. 


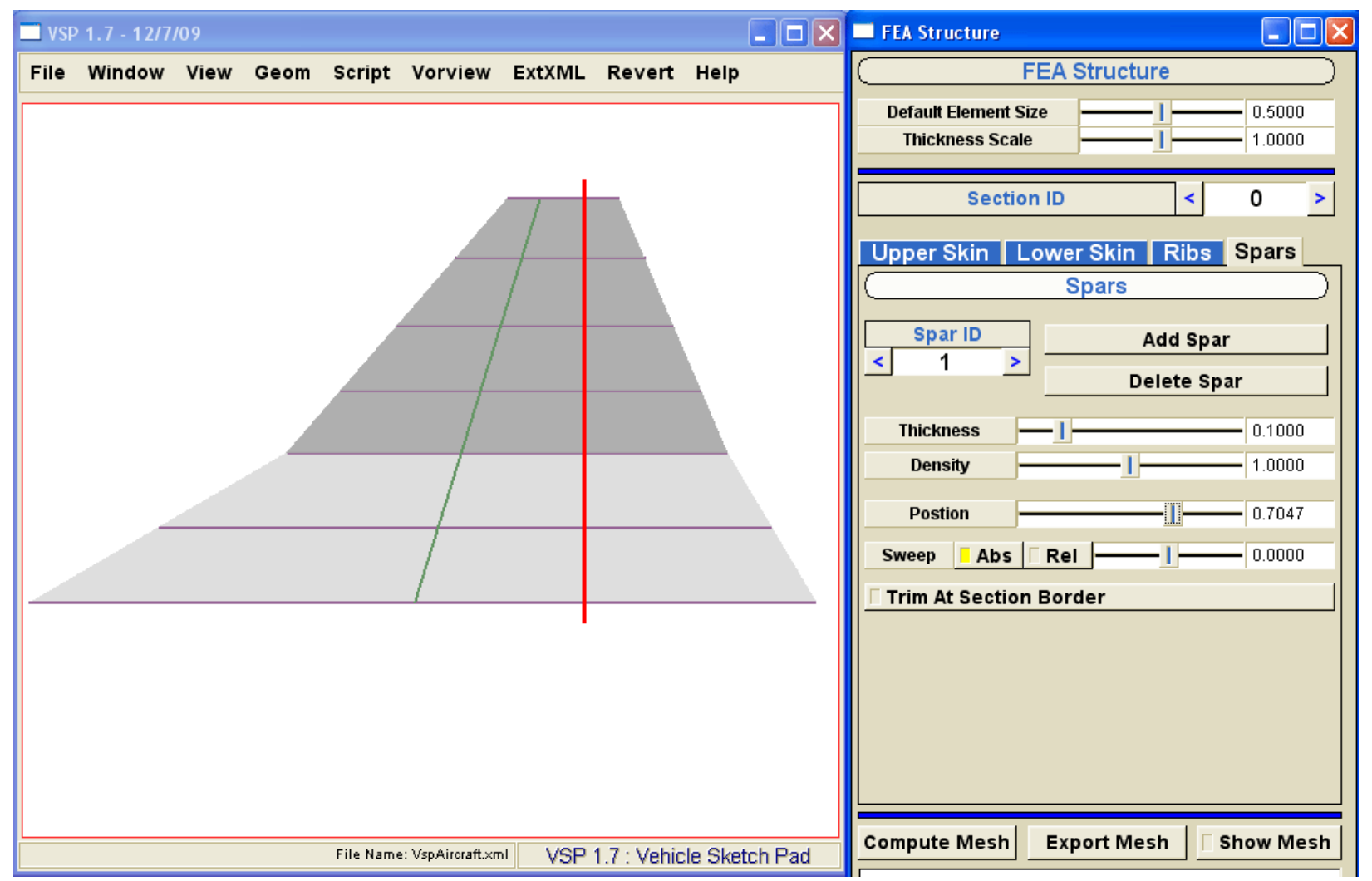

Figure 11. Structures control GUI for spars.

\section{Acknowledgements}

This work has been performed with the support of technical monitor Craig Nickol under NASA NRA NNX07AO14A. The structural layout improvements to VSP build on work done with the support of technical monitor Mark Moore under NASA STTR NNL06AA62P.

\section{References}

${ }^{1}$ Hahn, A., "Vehicle Sketch Pad: Parametric Geometry for Conceptual Aircraft Design," 48th AIAA Aerospace Sciences Meeting, Orlando, FL, Jan 4 - 7 2010, AIAA-2010-657.

${ }^{2}$ Fredericks, W., "Aircraft Conceptual Design Using Vehicle Sketch Pad," 48th AIAA Aerospace Sciences Meeting, Orlando, FL, January 4-7 2010, AIAA-2010-658.

${ }^{3}$ Snepp, D. K. and Pomeroy, R. C., "A geometry system for aerodynamic design," AHS, and ASEE, Aircraft Design, Systems and Operations Meeting, Saint Louis, MO, Sept 14-16 1987, pp. AIAA-1987-2902.

${ }^{4}$ Gloudemans, J. R., Davis, P. C., and Gelhausen, P. A., "A rapid geometry modeler for conceptual aircraft," 34 th Aerospace Sciences Meeting and Exhibit, AIAA-1996-52, Jan. 15-18 1996.

${ }^{5}$ La Rocca, G., Krakers, L., and van Tooren, M., "Development of an ICAD Generative Model for Blended Wing Body Aircraft Design," 9th AIAA/ISMO Symposium on Multidisciplinary Analysis and Optimisation, Atlanta, September 4-6 2002, AIAA 2002-5447.

${ }^{6}$ Sarakinos, S. S., Valakos, I. M., and Nikolos, I. K., "A software tool for generic parameterized aircraft design," Advances in Engineering Software, Vol. 38, No. 1, 2007, pp. 39 - 49.

${ }^{7}$ Athanasopoulos, M., Ugail, H., and Castro, G. G., "Parametric design of aircraft geometry using partial differential equations," Adv. Eng. Softw., Vol. 40, No. 7, 2009, pp. 479-486.

${ }^{8}$ AVID, "AVID PAGE (Parametric Aircraft Geometry Engine)," http://www.avidllc.biz/, 2010.

${ }^{9}$ Shewchuk, J. R., "Triangle: Engineering a 2D Quality Mesh Generator and Delaunay Triangulator," Applied Computational Geometry: Towards Geometric Engineering, edited by M. C. Lin and D. Manocha, Vol. 1148 of Lecture Notes in Computer Science, Springer-Verlag, May 1996, pp. 203-222, From the First ACM Workshop on Applied Computational Geometry.

${ }^{10}$ Wang, D., Hassan, O., Morgan, K., and Weatherill, N., "EQSM: An efficient high quality surface grid generation method 


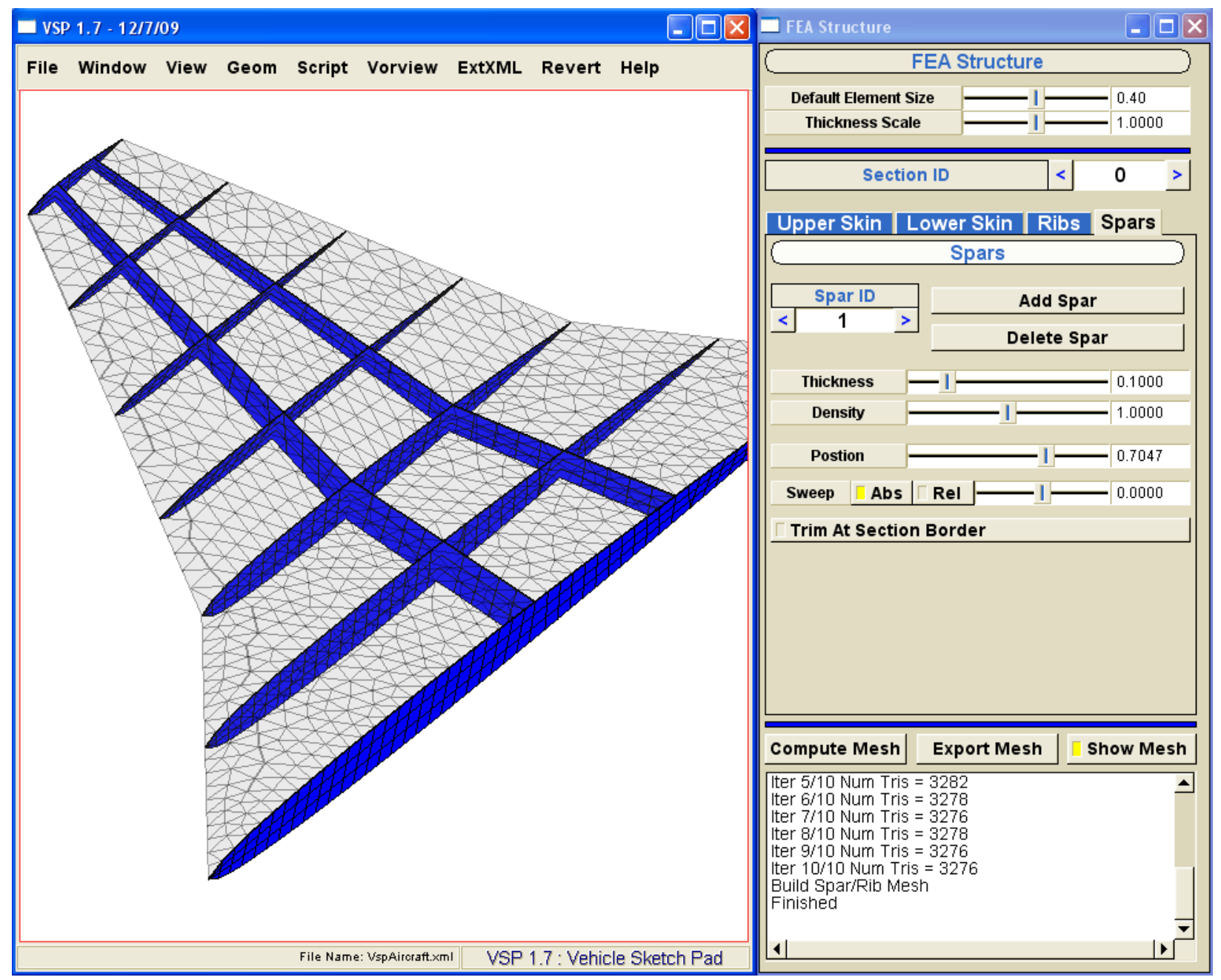

Figure 12. Structures control GUI for ribs. 


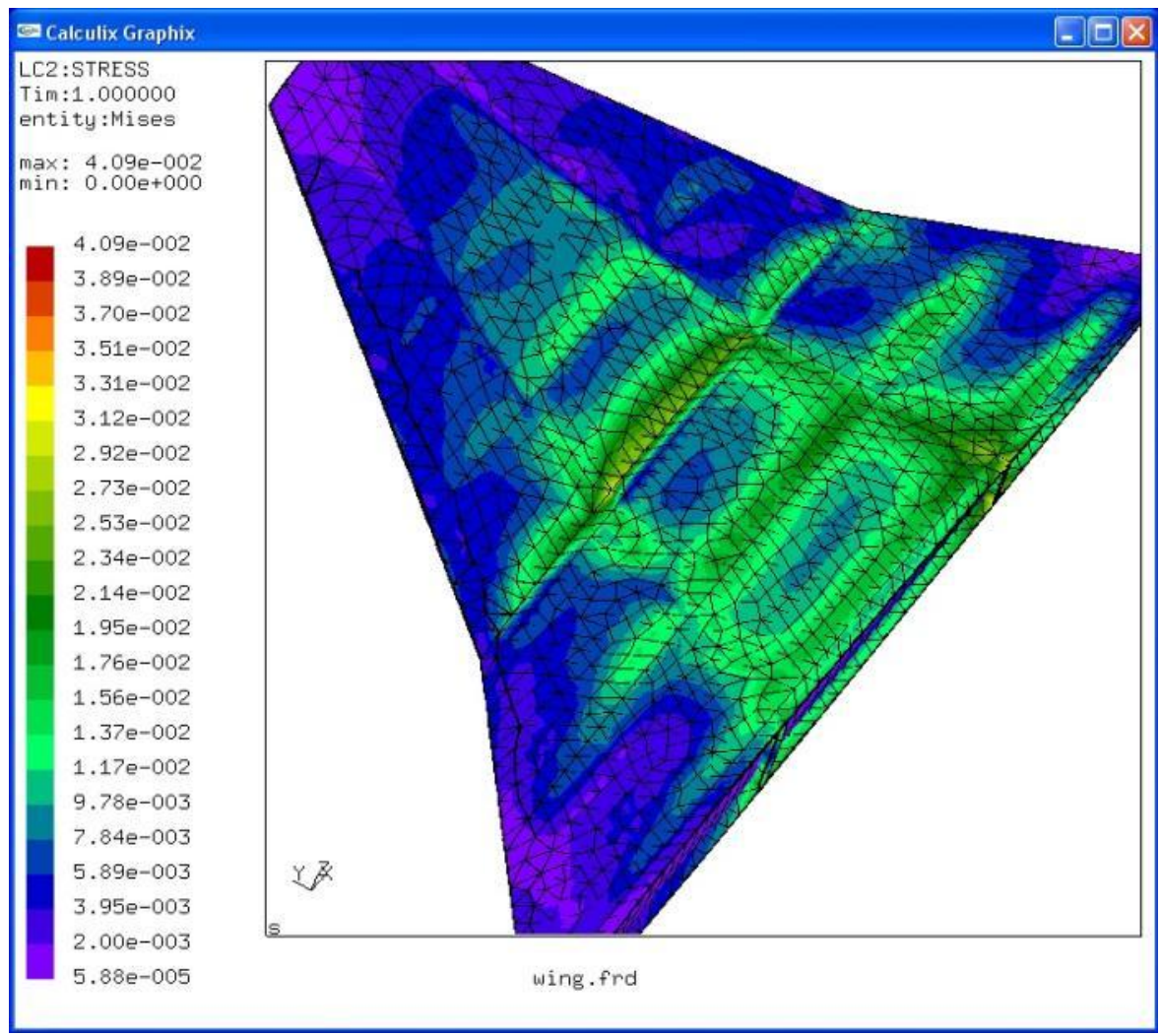

Figure 13. Calculix finite element solution.

based on remeshing," Computer Methods in Applied Mechanics and Engineering, Vol. 195, No. 41-43, 2006, pp. 5621 - 5633, John H. Argyris Memorial Issue. Part II.

${ }^{11}$ Houghton, E. G., Emnett, R. F., Factor, J. D., and Sabharwal, C. L., "Implementation of a divide-and-conquer method for intersection of parametric surfaces," Computer Aided Geometric Design, Vol. 2, No. 1-3, 1985, pp. 173 - 183. 